\title{
Estudo da composição e do rendimento do óleo essencial de tomilho (Thymus vulgaris $\mathrm{L}$.
}

\section{Study of composition and yield of Thymus vulgaris L. oil essential}

\author{
Elizabete Aparecida Ruzza Jakiemiu ${ }^{1 *}$; Agnes de Paula Scheer ${ }^{2}$; Juarez Souza de \\ Oliveira $^{3}$; Lilian Cristina Côcco ${ }^{4}$; Carlos Itsuo Yamamoto ${ }^{5}$; Cícero Deschamps ${ }^{6}$
}

\section{Resumo}

Embora extraído de uma mesma espécie vegetal, o rendimento e a composição química de um óleo essencial pode variar, significativamente, dependendo de fatores como a época de coleta, condições climáticas e de solo. Neste trabalho foram estudados o rendimento e a composição do óleo essencial de tomilho Thymus vulgaris L. cultivados na região de Curitiba - PR. O método de hidrodestilação em aparelho de Clevenger foi utilizado para a extração do óleo essencial das partes aéreas da planta fresca nos tempos de 1,2 e $3 \mathrm{~h}$. A identificação e a quantificação dos compostos foram efetuadas através de cromatografia gasosa e espectrometria de massas. Um total de 33 compostos foram identificados e quantificados. Dentre estes os majoritários foram timol, $p$-cimeno, carvacrol, $\gamma$-terpineno, linalol e borneol. O melhor tempo de extração para o rendimento do óleo essencial foi de $3 \mathrm{~h}$.

Palavras-chave: Óleos essenciais, Thymus vulgaris L., hidrodestilação

\begin{abstract}
The essential oil yield and quality on aromatic species is regulated by environmental conditions and vegetative development. The objective of this work was to evaluate the yield and composition of Thymus vulgaris L. essential oil from nearby Curitiba, PR. The essential oil was obtained from aerial fresh plants by hydrodistillation. The extraction was done during 1,2 and $3 \mathrm{~h}$. The components identification was performed by gas chromatography/mass spectrometry (GC/MS) and for quantification the equipment used was a gas chromatography with flame detector (GC/MS). There were identified and quantified thirty three components. The majority components were thymol, carvacrol and borneol. The best oil yield of $1.86 \%$ for fresh plants with aerial parts was obtained when used extraction time of $3 \mathrm{~h}$, however, no significant differences were found on components concentration for all the three extraction times.

Key words: Essential oil, Thymus vulgaris L., hydrodistillation
\end{abstract}

\footnotetext{
1 Mestrado em Tecnologia de Alimentos, Universidade Federal do Paraná. E-mail: elizabetejakiemiu@ufpr.br

2 Docente do Departamento de Engenharia Química, Universidade Federal do Paraná. E-mail: agnesps@ufpr.br

3 Docente do Departamento de Engenharia Química, Universidade Federal do Paraná. E-mail: juarzoli@ufpr.br

4 Docente do Departamento de Engenharia Química, Universidade Federal do Paraná. E-mail: liliancocco@ufpr.br

5 Docente do Departamento de Engenharia Química, Universidade Federal do Paraná. E-mail: ciyama@ufpr.br

6 Docente do Departamento de Fitotecnia, Universidade Federal do Paraná. E-mail: cícero@ufpr.br

* Autor para correspondência
} 
O tomilho (Thymus vulgaris L.) é uma planta da família Lamiaceae que compreende 150 gêneros, com cerca de 2800 espécies distribuídas em todo o mundo, sendo o maior centro de dispersão a região do Mediterrâneo. Muitas das espécies introduzidas no Brasil são plantas medicinais e produtoras de óleos essenciais, sendo utilizadas como condimentos ou como flores ornamentais. Dentre os gêneros cultivados da família Lamiaceae destacam-se várias espécies usadas como condimentos, tais como: sálvia (Salvia officinalis), manjericão (Ocimum basilicum), orégano (Origanum vulgaris L.), manjerona (Origanum majorana L.), entre outras (PORTE; GODOY, 2001).

Propriedades farmacológicas de diferentes extratos e dos óleos essenciais do tomilho foram estudadas detalhadamente e trouxeram contribuições significativas para as indústrias, principalmentecomo aditivos em alimentos e aplicações medicinais. A literatura menciona que o óleo essencial de tomilho possui atividades antimicrobianas carminativa e expectorante, atividades estas atribuídas ao timol e o carvacrol, componentes fenólicos do óleo, sendo o timol o mais potente. Atividades espasmolíticas bem como antioxidantes foram relatadas também para o extrato alcoólico da planta (HUDAIB et al., 2002). As atividades antifúngicas, pesticidas e antibacterianas do óleo essencial de tomilho foram demonstradas por diversos investigadores como Higes e Llorente (1996), Daferera, Ziogas e Polissiou (2000) e Bagamboula, Uyttendaele e Debevere (2004).

O rendimento e a composição dos extratos em diclorometano das folhas, das flores e das hastes do Thymus vulgaris L. que crescem no nordeste da Espanha foram estudados por Guillén e Manzanos (1998). O rendimento obtido das folhas e das flores é muito mais elevado do que aquele obtido das hastes, sendo encontrados $4,0 \%, 2,6 \%$ e $0,5 \%$, respectivamente.
Outros estudos fitoquímicos investigaram a composição do óleo essencial do Thymus vulgaris L. de fontes e de genótipos diferentes, bem como a sua variação em estações diferentes e durante o ciclo vegetativo da planta (HUDAIB et al., 2002). Avaliações da composição do óleo extraído de diferentes partes da planta em condições diversas de cultivo, e/ou de armazenamento também têm sido relatadas (VENSKUTONIS; POLL; LARSEN, 1996).

O presente trabalho teve por objetivo avaliar o rendimento do óleo essencial de tomilho obtido de plantas frescas bem como identificar e quantificar os compostos presentes no mesmo.

As amostras de planta fresca de tomilho (Thymus vulgaris L., família Lamiaceae), cultivadas na região de Curitiba-PR foram coletadas no mês de julho de 2007. Após a colheita foram divididas em dois grupos. No primeiro grupo, foram mantidas as hastes e as folhas, denominadas neste estudo de fresca haste, e no segundo trabalharam-se somente com as folhas sendo denominadas frescas folhas. Em ambos os grupos as amostras foram homogeneizadas, pesadas e, em seguida, foi efetuada a extração.

A identificação botânica das plantas foi realizada por meio de comparação com exsicata depositada no Museu Botânico Municipal de Curitiba sob registro $N^{o}$ 218653, efetuada pelo botânico responsável Gerdt Gunther Hatschbach.

Foram pesados em triplicata aproximadamente $100 \mathrm{~g}$ de amostra de tomilho (Thymus vulgaris L.) e transferidos para um balão volumétrico ao qual se adicionou $1 \mathrm{~L}$ de água destilada. Acoplou-se ao balão o aparelho de Clevenger, iniciando-se a hidrodestilação. As amostras deóleo essencial obtidas em diferentes tempos de extração (1, 2 e 3 h) foram transferidas para tubos ependorf e posteriormente analisadas pela técnica de cromatografia a gás para a quantificação e, cromatografia a gás acoplada a espectrometria de massas, para a identificação dos constituintes. 
O rendimento do óleo essencial foi expresso em \% volume/massa, ou seja, $\mathrm{mL}$ de óleo essencial, por gramas (g) de material vegetal seco (FARMACOPÉIA BRASILEIRA, 1988; FABROWSKI, 2002). A secagem das amostras de $20 \mathrm{~g}$ de massa verde foi feita em triplicata em estufa com circulação de ar à temperatura de $65^{\circ} \mathrm{C}$ até massa constante (FARMACOPÉIA BRASILEIRA, 2000).

$\mathrm{O}$ equipamento e as condições operacionais utilizadas para a quantificação dos componentes do óleo essencial de tomilho foram as seguintes: cromatográfo a gás Varian, modelo CP 3800 com detector FID (CG-FID). Utilizou-se coluna capilar Chrompack de sílica fundida CP-SIL 8 CB, 0,25mm de diâmetro interno, $30 \mathrm{~m}$ de comprimento e $0,25 \mu \mathrm{m}$ de filme líquido. A temperatura do injetor foi de $250^{\circ} \mathrm{C}$, "split" 1:200. A quantidade de amostra injetada foi igual a 1,0 $\mu \mathrm{L}$. O gás de arraste utilizado foi o gás hélio com a pressão da coluna de 30psi e gás de "make up" ar sintético, nitrogênio e hidrogênio. A temperatura do detector FID foi de $300^{\circ} \mathrm{C}$, com programação de temperatura do forno a temperatura inicial de $60^{\circ} \mathrm{C}$ e elevação de temperatura a $90^{\circ} \mathrm{C}$ na razão de $3^{\circ} \mathrm{C}$ por min, permanecendo por $5 \mathrm{~min}$; elevação de temperatura a $140^{\circ} \mathrm{C}$ na razão de $3^{\circ} \mathrm{C}$ por min, elevação de temperatura a $240^{\circ} \mathrm{C}$ na razão de $30^{\circ} \mathrm{C}$ por min, permanecendo por $5 \mathrm{~min}$.

As condições operacionais para aquisição do espectro de massas para a identificação dos constituintes do óleo essencial de tomilho foram as seguintes: cromatográfo a gás acoplado a um detector de massas (CG-EM) Varian com "íon trap", modelo CP 3.800/Saturn 2000. Utilizou-se coluna capilar Chrompack de sílica fundida CP-SIL 8 CB, $0,25 \mathrm{~cm}$ de diâmetro interno, $30 \mathrm{~m}$ de comprimento e $0,25 \mu \mathrm{m}$ de filme líquido. A temperatura do injetor foi de $250^{\circ} \mathrm{C}$ e "split" 1:300. A quantidade de amostra injetada variou de 0,2 a $0,5 \mu \mathrm{L}$. O gás de arraste utilizado foi o gás hélio a $1 \mathrm{~mL} / \mathrm{min}$ constante. A temperatura do "transfer line" foi de $250^{\circ} \mathrm{C}$, a temperatura do "manifold" foi de $80^{\circ} \mathrm{C}$ e a temperatura do "ion trap" de $150^{\circ} \mathrm{C}$ e modulação axial de 4V. A intensidade da ionização foi de $70 \mathrm{eV}$ e o modo de ionização utilizado foi por impacto de elétrons. A programação de temperatura do forno foi: temperatura inicial de $60^{\circ} \mathrm{C}$, elevação de temperatura a $90^{\circ} \mathrm{C}$ na razão de $3^{\circ} \mathrm{C}$ por min permanecendo por 5 min; elevação de temperatura a $140^{\circ} \mathrm{C}$ na razão de $3^{\circ} \mathrm{C}$ por min; elevação de temperatura a $240^{\circ} \mathrm{C}$ na razão de $30^{\circ} \mathrm{C}$ por min permanecendo por $5 \mathrm{~min}$. Tempo total de corrida de $40 \mathrm{~min}$.

O espectro de massas de cada um dos componentes do óleo essencial de tomilho foi analisado e comparado aos espectros contidos no acervo das bibliotecas Saturn (CG-EM versão 5.51), Nist (98 MS, versão 1.7) e Adams (1995). Os dados de rendimento e composição foram submetidos à análise de variância e suas médias comparadas pelo teste de Tukey com 5\% de probabilidade.

O rendimento do óleo essencial de tomilho apresentado na (Tabela 1) variou entre 1,04\% $(\mathrm{mL} / 100 \mathrm{~g}$, base seca) para $1 \mathrm{~h}$ a $1,86 \%(\mathrm{~mL} / 100 \mathrm{~g}$, base seca) para $3 \mathrm{~h}$ na planta fresca haste. Este rendimento está de acordo com a ISO 6754:1996, que especifica a porcentagem mínima de óleos voláteis para o Thymus vulgaris L. em 1,00\% $(\mathrm{mL} / 100 \mathrm{~g}$, base seca). Usando as partes aéreas das plantas frescas de Thymus vulgaris L., Hudaib et al. (2002) obtiveram um rendimento de $0,15 \%$ (v/m) para plantas velhas, porém para plantas novas foi de $1,20 \%(\mathrm{v} / \mathrm{m})$. Ozcan e Chalchat (2004) obtiveram rendimento de $1,57 \%(\mathrm{v} / \mathrm{m})$ sem mencionar a idade das plantas e nas mesmas condições de ensaio usadas neste trabalho.

Observou-se neste estudo que, no final do período de $3 \mathrm{~h}$, a taxa de extração do óleo foi muito pequena, sendo assim, não foram testados tempos superiores e adotou-se o tempo máximo de 3h, utilizado na literatura (OZCAN; CHALCHAT, 2004). 
Nas plantas frescas (haste e folhas) os compostos majoritários foram o timol com 54,00 a 57,50\% de concentração para fresca haste, e 50,43 a 55,00\% de concentração para frescas folhas, seguido pelo p-cimeno com 12,00 a $15,00 \%$ e 17,00 a $21,00 \%$ respectivamente. A concentração do $\gamma$-terpineno variou de 6,00 a $7,00 \%$ para a planta fresca haste e de 5,00 a 7,00\% para planta fresca folhas, como mostrado na tabela 2. A concentração dos compostos carvacrol e borneol variou de $2,35 \%$ a $4,01 \%$ e de $1,59 \%$ a $3,40 \%$ respectivamente.

Pode-se observar na (Tabela 2) que a variação da concentração com o aumento do tempo de extração não seguiu o padrão uniforme. Dependendo do composto houve um aumento ou uma diminuição da concentração, portanto o estudo deve ser aprofundado tanto na parte experimental quanto na análise estatística das variações.

Nas condições experimentais deste trabalho conclui-se que o tempo de extração por hidrodestilação que resultou no melhor rendimento de óleo essencial foi de $3 \mathrm{~h}$. Foram identificados 33 compostos, sendo majoritários o timol, o $p$-cimeno e o $\gamma$-terpineno.

Tabela 1. Rendimento médio em porcentagem volume/massa por tempo de extração e tipo de planta

\begin{tabular}{cccc}
\hline AMOSTRAS & \multicolumn{3}{c}{ TEMPO DE EXTRAÇÃO } \\
\hline & $1 \mathrm{~h}$ & $2 \mathrm{~h}$ & $3 \mathrm{~h}$ \\
\cline { 2 - 4 } Planta fresca haste & $1,04^{\mathrm{cB}}$ & $1,32^{\mathrm{bB}}$ & $1,86^{\mathrm{aA}}$ \\
Planta fresca folhas & $1,52^{\mathrm{cA}}$ & $1,68^{\mathrm{bA}}$ & $1,78^{\mathrm{aA}}$ \\
\hline
\end{tabular}

* Letras minúsculas iguais na horizontal não diferem significativamente quanto ao rendimento por hora pelo teste de Tukey, com $95 \%$ de significância.

* Letras maiúsculas iguais na vertical não diferem significativamente quanto ao rendimento por planta pelo teste de Tukey, com $95 \%$ de significância. 
Tabela 2. Identificação e quantificação dos compostos do óleo essencial de tomilho extraídos por hidrodestilação da planta fresca.

\begin{tabular}{|c|c|c|c|c|c|c|c|c|c|}
\hline & \multirow[t]{3}{*}{ IR } & \multirow[t]{3}{*}{ IRL } & \multirow[t]{3}{*}{ COMPOSTO } & \multicolumn{6}{|c|}{$\begin{array}{c}\text { TIPO DE AMOSTRA / TEMPO DE EXTRAÇÃO / } \\
\% \text { DE COMPOSTOS }\end{array}$} \\
\hline & & & & \multicolumn{3}{|c|}{ Fresca haste } & \multicolumn{3}{|c|}{ Fresca folhas } \\
\hline & & & & $1 \mathrm{~h}$ & $2 \mathrm{~h}$ & $3 \mathrm{~h}$ & $1 \mathrm{~h}$ & $2 \mathrm{~h}$ & $3 \mathrm{~h}$ \\
\hline 1 & 918 & 926 & Tricicleno & 0,93 & 0,85 & 0,39 & 0,69 & 0,83 & 0,43 \\
\hline 2 & 928 & 939 & $\alpha$-pineno & 0,64 & 0,49 & 0,25 & 0,53 & 0,51 & 0,27 \\
\hline 3 & 944 & 953 & Canfeno & 0,63 & 0,43 & 0,28 & 0,65 & 0,76 & 0,58 \\
\hline 4 & 967 & 976 & Sabineno & $\operatorname{Tr}$ & $\operatorname{Tr}$ & $\operatorname{Tr}$ & $\operatorname{Tr}$ & $\operatorname{Tr}$ & $\operatorname{Tr}$ \\
\hline 5 & 973 & 980 & $\beta$-pineno & 0,80 & 0,88 & 1,00 & 1,00 & 1,48 & 0,92 \\
\hline 6 & 984 & 991 & Mirceno & 1,23 & 1,09 & 0,79 & 1,00 & 1,45 & 0,54 \\
\hline 7 & 1014 & 1018 & $\alpha$-Terpineno & 0,45 & 0,51 & 0,46 & 5,64 & 6,78 & 5,77 \\
\hline 8 & 1021 & 1026 & $p$-Cimeno & 15,49 & 12,86 & 13,92 & 18,60 & 21,17 & 17,92 \\
\hline 9 & 1025 & 1031 & Limoneno & 0,31 & 0,28 & 0,16 & 0,29 & 0,37 & 0,22 \\
\hline 10 & 1027 & 1031 & $\beta$-felandreno & 0,10 & 0,13 & 0,14 & 0,08 & 0,36 & 0,21 \\
\hline 11 & 1029 & 1033 & Eucaliptol & 0,50 & 0,42 & 0,34 & 0,60 & 0,81 & 0,49 \\
\hline 12 & 1054 & 1062 & $\gamma$-Terpineno & 6,90 & 7,20 & 6,20 & 5,64 & 7,17 & 5,71 \\
\hline 13 & 1083 & 1088 & Terpinoleno & 0,01 & 0,02 & 0,03 & 0,03 & 0,09 & 0,05 \\
\hline 14 & 1099 & 1098 & Linalol & 1,80 & 1,74 & 2,06 & 2,96 & 4,10 & 2,42 \\
\hline 15 & 1141 & 1143 & Cânfora & 0,66 & 0,46 & 0,55 & 0,57 & 0,69 & 0,35 \\
\hline 16 & 1167 & 1165 & Borneol & 1,74 & 1,59 & 1,97 & 2,86 & 3,40 & 2,17 \\
\hline 17 & 1175 & 1177 & Terpin-4-ol & 0,55 & 0,60 & 0,64 & 0,56 & 0,56 & 0,32 \\
\hline 18 & 1194 & 1189 & $\alpha$-Terpineol & 0,27 & 0,20 & 0,23 & 0,32 & 0,42 & 0,17 \\
\hline 19 & 1227 & 1233 & Formato de Isobornila & 0,15 & 0,58 & 1,22 & 0,18 & 0,35 & 0,26 \\
\hline 20 & 1239 & 1244 & Eter Metil Carvacrol & $\operatorname{Tr}$ & $\operatorname{Tr}$ & $\operatorname{Tr}$ & $\operatorname{Tr}$ & $\operatorname{Tr}$ & $\operatorname{Tr}$ \\
\hline 21 & 1245 & 1246 & Carvotanacetona & $\operatorname{Tr}$ & $\operatorname{Tr}$ & $\operatorname{Tr}$ & $\operatorname{Tr}$ & $\operatorname{Tr}$ & $\operatorname{Tr}$ \\
\hline 22 & 1283 & 1285 & Acetato de Bornila & 0,37 & 0,44 & 0,41 & 0,47 & 0,51 & 0,29 \\
\hline 23 & 1293 & 1290 & Timol & 54,89 & 57,58 & 55,33 & 50,43 & 55,25 & 52,67 \\
\hline 24 & 1301 & 1298 & Carvacrol & 3,18 & 3,43 & 3,41 & 3,54 & 4,01 & 2,35 \\
\hline 25 & 1354 & 1356 & Eugenol & $\operatorname{Tr}$ & $\operatorname{Tr}$ & $\operatorname{Tr}$ & $\operatorname{Tr}$ & $\operatorname{Tr}$ & $\operatorname{Tr}$ \\
\hline 26 & 1413 & 1404 & Cariofileno & 1,05 & 1,46 & 1,90 & 0,89 & 0,88 & 0,36 \\
\hline 27 & 1441 & $*$ & $\begin{array}{l}\text { Orto-metoxi-alfa-alfa- } \\
\text { dimetil-benzil-alcool }\end{array}$ & $\operatorname{Tr}$ & $\operatorname{Tr}$ & $\operatorname{Tr}$ & $\operatorname{Tr}$ & $\operatorname{Tr}$ & $\operatorname{Tr}$ \\
\hline 28 & 1512 & 1513 & $\gamma$-Cadineno & $\operatorname{Tr}$ & $\operatorname{Tr}$ & $\operatorname{Tr}$ & $\operatorname{Tr}$ & $\operatorname{Tr}$ & $\operatorname{Tr}$ \\
\hline 29 & 1517 & 1524 & $\delta$-Cadineno & $\operatorname{Tr}$ & $\operatorname{Tr}$ & $\operatorname{Tr}$ & $\operatorname{Tr}$ & $\operatorname{Tr}$ & $\operatorname{Tr}$ \\
\hline 30 & 1521 & 1520 & Miristicina & $\operatorname{Tr}$ & $\operatorname{Tr}$ & $\operatorname{Tr}$ & $\operatorname{Tr}$ & $\operatorname{Tr}$ & $\operatorname{Tr}$ \\
\hline 31 & 1578 & 1576 & Espatulenol & $\operatorname{Tr}$ & $\operatorname{Tr}$ & $\operatorname{Tr}$ & $\operatorname{Tr}$ & $\operatorname{Tr}$ & $\operatorname{Tr}$ \\
\hline 32 & 1644 & 1645 & $\alpha$-Muurolol & $\operatorname{Tr}$ & $\operatorname{Tr}$ & $\operatorname{Tr}$ & $\operatorname{Tr}$ & $\operatorname{Tr}$ & $\operatorname{Tr}$ \\
\hline 33 & 1657 & 1653 & $\alpha$-Cadinol & $\operatorname{Tr}$ & $\operatorname{Tr}$ & $\operatorname{Tr}$ & $\operatorname{Tr}$ & $\operatorname{Tr}$ & $\operatorname{Tr}$ \\
\hline
\end{tabular}

** O teste de Tukey não apresentou diferença significativa ao nível de 95\% de significância na concentração dos compostos entre 1,2 e 3 horas.

NOTA: IR - Índice de retenção; Tr - Traços, concentração < 0,001; * - Índice de retenção não encontrado na literatura. 


\section{Agradecimentos}

Ao Laboratório de Combustíveis Automotivos - LACAUT, pelas análises de cromatografia e espectrometria de massas; Ao CNPQ pelo apoio financeiro.

\section{Referências}

ADAMS, R. P. Identification of essencial oil components by gás Chormatography Mass Spectroscopy. Carol Strean, Illinois, USA: Allured Publishing Corporation, 1995. 469 p.

BAGAMBOUla, C. F.; UYTTENDAELE, M.; DEBEVERE, J. Inhibitory effect of thyme and basil essential oils, carvacrol, thymol, estragol, linalool and p-cymene towards Shigella sonnei and S. flexneri. Food Micro., Ghent, Belgium, v. 21, n. 1, p. 33-42, 2004.

DAFERERA, D. J.; ZIOGAS, B. N.; POLISSIOU, M. G. CG/EM analysis of essential oils from some Greek aromatic plants and their fungitoxicity on Penicillium digitatum. J. Agric. Food Chem., Athens, Greece, v. 48, n. 6, p. 2576-2581, 2000.

FABROWSKI, F. J. Eucaliptus smithii R. T. BAKER (Myrtaceae) como espécie produtora de óleo essencial no sul do Brasil. 2002. Tese (Doutorado em Engenharia Florestal) - Centro de Ciências Agrárias. Universidade Federal do Paraná, Curitiba.

FARMACOPÉIA BRASILEIRA. 4. ed. São Paulo: Atheneu, 1988. 4 ed. São Paulo: Atheneu, 2000.
GUILLÉN, M. D.; MANZANOS, M. J. Study of the composition of the different parts of a Spanish Thymes vulgaris L. Plant. Food Chemistry, Vitoria, Spain, v. 63, n. 3, p. 373-383, 1998.

HIGES, M.; LLORENTE, J. Ensayo de la eficacia del timol en el control de la varroosis de Apis mellifera en colmenas en producción. Actas del II Congreso de la SEAE. Agricultura ecológica y desarrollo rural. Navarra, p. 205-210, 1996.

HUDAIB, M.; SPERONI, E.; PIETRA, A. M. D.; CAVRINI, V. CG/EM evaluation of thyme (Thymus Vulgaris L.) oil composition and variations during the vegetative cycle. Journal of Pharmaceutical and Biomedical Analysis, Bologna, Italy, v. 29, n. 4, p. 691700, 2002.

NIST 98, Library. Search v. 1.7. lnk: Database in saturn and nist format. [S.1.]: [s.n.], 2001.

OZCAN, M.; CHALCHAT, J. Aroma profile of thymus vulgaris L. growing wild in Turkey. Bulg. J. Plant Physiol, Turkey, v. 30, n. 3/4, p. 68-73, 2004.

PORTE, A.; GODOY, R. L. O. Alecrim (Rosmarinus Officinalis L.): propriedades antimicrobiana e química do óleo essencial. Boletim CEPPA, Curitiba, v. 19, n. 2, p. 193-210, jul./dez. 2001.

VENSKUTONIS, R.; POLL, L.; LARSEN, M. Influence of drying and irradiation on the composition of volatile compounds of thyme (Thymus vulgaris L.). Flavour and Fragance Journal, Lithuania, v. 11, n. 2, p. 123-128, 1996. 